\title{
Propagation delay time estimation by neural network using urban environment parameters
}

\author{
Koyo Tategami $^{1 \text {, a) }}$, Mitoshi Fujimoto ${ }^{1}$ Koshiro Kitao $^{2}$, \\ Minoru Inomata ${ }^{2}$, Satoshi Suyama ${ }^{2}$, and Yasuhiro Oda ${ }^{2}$ \\ ${ }^{1}$ Graduate School of Engineering, University of Fukui, \\ 3-9-1 Bunkyo, Fukui 910-8507, Japan \\ ${ }^{2}$ NTT DOCOMO, INC., \\ 3-6 Hikarino-oka, Yokosuka-shi, Kanagawa 239-8536, Japan \\ a) tategami@wireless.fuis.u-fukui.ac.jp
}

\begin{abstract}
In the $5^{\text {th }}$ generation mobile communication system, introduction of small cells using low-height base station is being examined. Small cells are installed in urban street cell environments where mobiles are densely populated. Therefore, actual measurement and complicated modeling of radio wave propagation are necessary to grasp the propagation characteristics. In this paper, a simple propagation delay estimation method is proposed. In the proposed method, neural network using urban area structure parameters is applied. It is shown that the propagation delay time can be easily estimated by the proposed method without considering moving objects and trees.
\end{abstract}

Keywords: 5G mobile communication system, urban street cell, neural network, propagation delay time estimation

Classification: Antennas and Propagation

\section{References}

[1] NTT DOCOMO, "DOCOMO 5G White Paper, 5G Radio Access: Requirements, Concept and Technologies," July 2014.

[2] K. Tategami, M. Fujimoto, K. Kitao, and T. Imai, "Angle of arrival characteristics at $20 \mathrm{GHz}$ band in LOS urban area street cell environment," Proc. 2019 IEEE International Workshop on Electromagnetics, PS-7, Sep. 2019. DOI: 10.1109/iwem.2019.8887950

[3] M. Inomata, T. Imai, K. Kitao, Y. Okumura, M. Sasaki, and Y. Takatori, "Radio propagation prediction method using point cloud data based on hybrid of raytracing and effective roughness model in urban environments," IEICE Trans. Commun., vol. E102-B, no. 1, pp. 51-62, Jan. 2019. DOI: 10.1587/transcom. 2017EBP3436

[4] M. Sasaki, M. Inomata W. Yamada, N. Kita, T. Onizawa, M. Nakatsugawa, K. Kitao, and T. Imai, "Path loss model considering blockage effects of traffic signs up to $40 \mathrm{GHz}$ in urban microcell environments," IEICE Trans. Commun., vol. E101-B, no. 8, pp. 1891-1902, Aug. 2018. DOI: 10.1587/transcom. 
[5] S.B. Kotsiantis, "Supervised machine learning: a review of classification techniques," Informatica, vol. 31, pp. 249-268, July 2007.

[6] K. Saito, Y. Jin, C. Kang, J. Takada, and J. Leu, “Two-step path loss prediction by artificial neural network for wireless service area planning," IEICE Commun. Express, vol. 8, no. 12, pp. 611-616, Sep. 2019. DOI: 10.1587/comex. 2019GCL0038

[7] S.N. Livieratos and P.G. Cottis, "Rain attenuation along terrestrial millimeter wave links: a new prediction method based on supervised machine learning," IEEE Access, vol. 7, pp. 138745-138756, Oct. 2019. DOI: 10.1109/ ACCESS.2019.2939498

[8] R. Rojas, Neural Networks: A Systematic Introduction, Springer-Verlag, Berlin, 1996.

\section{Introduction}

In the 5th generation mobile communication systems, introduction of small cells using low-height base station is being examined. The major installation environments of small cells are urban street cell environments where pedestrians and vehicles are densely populated [1]. In order to understand the propagation characteristics in such environments, it is necessary to analyze the data obtained by field measurements [2] or to perform simulation analysis by complex modeling of moving objects and trees $[3,4]$.

In this paper, a simple propagation delay estimation method is proposed. In the proposed method, the delay profile measured by the channel sounder is applied to the trainings data of neural network $[5,6,7]$. And also the urban area structure and the parameters calculated from them are applied as the inputs data of the neural network. This paper is organized as follows. The proposed propagation delay time estimation method is explained in Section 2. The measurement environments and specifications are explained in Section 3. Comparison of measured results and estimated results is explained in Section 4. The conclusion is provided in Section 5.

\section{Propagation delay time estimation by neural network}

\subsection{Outline of the proposed estimation method}

In the proposed method, neural network is used to realize simple propagation delay time estimation. The process of the proposed method is as follows.

Step1. Model the simplified street model on the urban area.

Step2. Calculate delay time using the simplified street model.

Step3. Learn the neural network model. The input data for the learning is dimension of the simplified street model (in step1) and calculated delay times (in step2). The training data for the learning is the measured delay profile.

Step4. Estimate the delay profile by inputting unmeasured parameters into the learned model in step3.

In order to estimate the propagation delay time without considering moving objects and trees, the actually measured delay profile is used as training data. Since the measured delay profile reflects the effects of moving objects and trees, these effects are also reflected in the model obtained by learning. Also, by using the results 

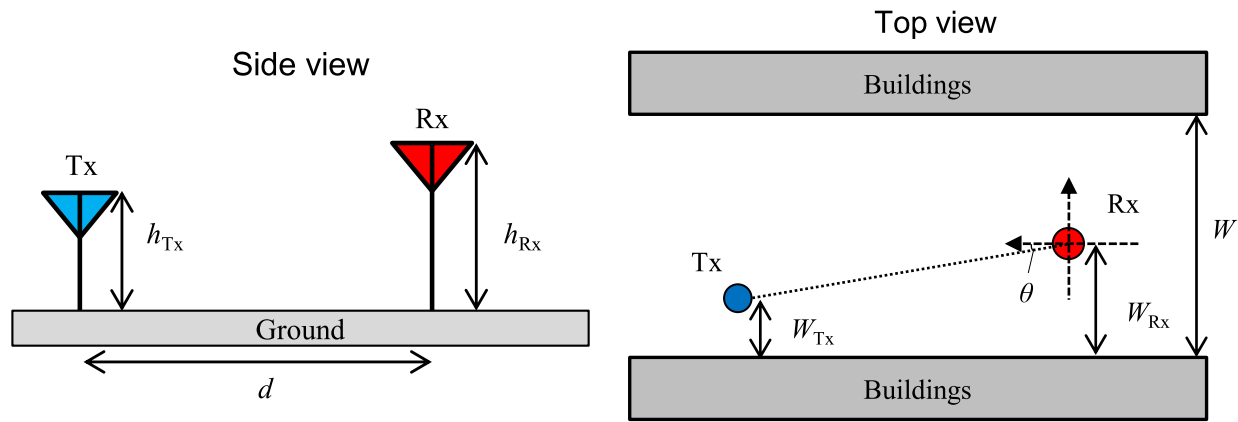

(a) Modeling the measurement area into the simplified street model

(b) Input parameters

\begin{tabular}{|c|c|c|}
\hline \multirow{6}{*}{$\begin{array}{l}\text { Dimension } \\
{[\mathrm{m}]}\end{array}$} & $h_{\mathrm{Tx}}$ & Transmitting station height \\
\hline & $h_{\mathrm{Rx}}$ & Base station height \\
\hline & $d$ & Distance between the transmitting station and the base station \\
\hline & $W$ & Road width \\
\hline & $W_{\mathrm{Tx}}$ & Distance between the transmitting station and the nearest building \\
\hline & $\frac{1 \Lambda}{W_{\mathrm{Rx}}}$ & Distance between the base station and the nearest building \\
\hline \multirow{3}{*}{$\begin{array}{l}\text { Delay time } \\
\quad[\mathrm{ns}]\end{array}$} & $\tau_{1}$ & Delay time of direct wave \\
\hline & $\tau_{2}$ & $\begin{array}{l}\text { Delay time of the reflected wave } \\
\text { on the wall of the building near the transmitting station }\end{array}$ \\
\hline & $\tau_{3}$ & $\begin{array}{c}\text { Delay time of reflected wave } \\
\text { on the wall of the building distant from the transmitting station }\end{array}$ \\
\hline
\end{tabular}

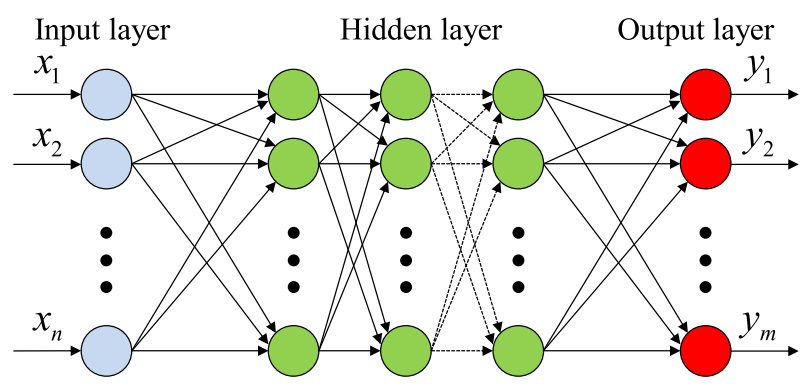

(c) Neural network model

Fig. 1. Proposed propagation delay time estimation method by the neural network

measured at many points for learning, it is possible to estimate the results at points where no measurement is performed.

\subsection{Input parameters}

First, the urban area is modeled to the simplified street model consisting of ground and buildings as shown in Fig. 1 (a). This model is constructed using the dimension parameters shown in Fig. 1 (b) of the actual measurement environment. The dimension parameters can be easily obtained. The delay time shown in Fig. 1 (b) is calculated using the dimension parameter of the simplified street model. The path length of the radio waves is calculated geometrically from the simplified street model, and the delay time is calculated. The delay time $\tau_{1}$ is the direct wave, the delay time $\tau_{2}$ is the reflected wave from the building near the transmitting station, and the delay time $\tau_{3}$ is the reflected wave from the building far from the transmitting station. Besides the delay time from buildings, the delay time of the ground 
reflection wave can be calculated, but the delay time difference between the direct wave and the ground reflection wave is very small. This means that the delay time of the ground reflection wave has a small role as a feature. Therefore, it is not used as an input parameter. The respective delay times are expressed by the following equations.

$$
\begin{aligned}
& \tau_{1}=\frac{\sqrt{d^{2}+\left(h_{\mathrm{Rx}}-h_{\mathrm{Tx}}\right)^{2}}}{c} \times 10^{9} \\
& \tau_{2}=\frac{\sqrt{\left(2 W_{\mathrm{Tx}}+d \sin |\theta|\right)^{2}+(d \cos |\theta|)^{2}+\left(h_{\mathrm{Rx}}-h_{\mathrm{Tx}}\right)^{2}}}{c} \times 10^{9} \\
& \tau_{3}=\frac{\sqrt{\left(2\left(W-W_{\mathrm{Tx}}\right)-d \sin |\theta|\right)^{2}+(d \cos |\theta|)^{2}+\left(h_{\mathrm{Rx}}-h_{\mathrm{Tx}}\right)^{2}}}{c} \times 10^{9}
\end{aligned}
$$

Here, $\theta$ is the angle of the transmitting station with respect to the receiving station, and $c$ is the speed of light.

These parameters are normalized by the maximum value per each environment and input to the neural network model in Fig. 1 (c).

\subsection{Output parameters}

The output layer of the neural network shown in Fig. 1 (c) corresponds to the delay bin, and outputs the probability value that a wave arrives within each delay from each neuron. Since the delay profile used for learning is normalized by the maximum value per delay profile obtained for each environment, the value of the delay profile ranges from 0 to 1 . This relative power can be regarded as a probability value. Therefore, the output probability value corresponds to the relative power.

\section{Measurement environment and specifications}

Figures 2 (a) and 2 (b) show the measurement area of the actual measurement data, Fig. 2 (c) shows a schematic diagram of the measurement system, and Fig. 2 (d) shows the measurement specifications. The measurement environments were urban street cell environments in which buildings lined up on both sides of the road. Only the LOS measurements are discussed in this paper. In addition, there were running and parking vehicles on the road. Also, there were trees and subway entrances, lights, pedestrians, etc. on the sidewalk.

The measurement was conducted in an uplink, and the transmitting station (Tx) transmitted an OFDM signal with a bandwidth of about $45 \mathrm{MHz}$ in a $20 \mathrm{GHz}$ band using a sleeve antenna. The transmission power was $30 \mathrm{dBm}$. On the base station (receiving station) [BS (Rx)], signals were received using a $256(16 \times 16)$ elements planar patch array antenna. The base station antenna height was $5 \mathrm{~m}$. The transmitting station antenna heights were $1.5 \mathrm{~m}$ or $2.5 \mathrm{~m}$ in Kayaba, and only $2.5 \mathrm{~m}$ in Hatchobori. When the transmitting station was installed on the sidewalk, its height was $1.5 \mathrm{~m}$, and when it was installed on the roadway, its height was $2.5 \mathrm{~m}$. The measurements were conducted at fixed-point. At each position of the transmitting station, data was recorded 30 times at 1 -second intervals on the receiving station side, 


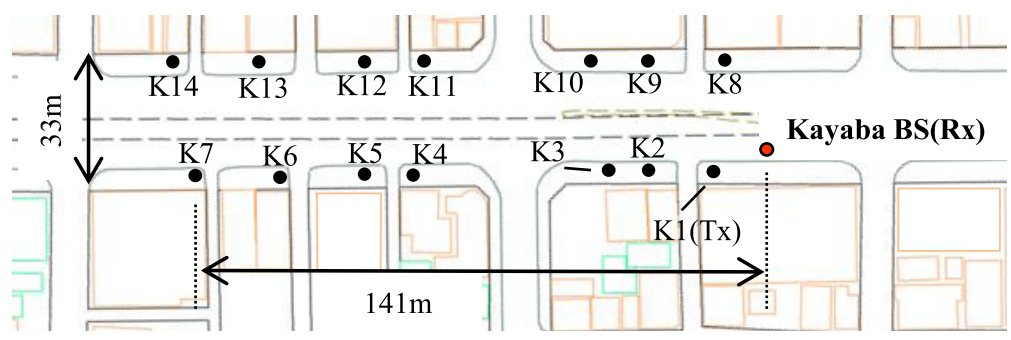

(a) Measurement area in Kayaba

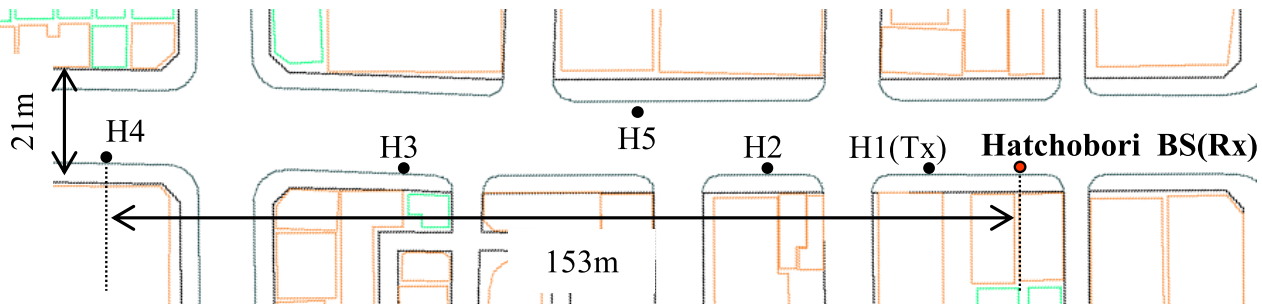

(b) Measurement area in Hatchobori

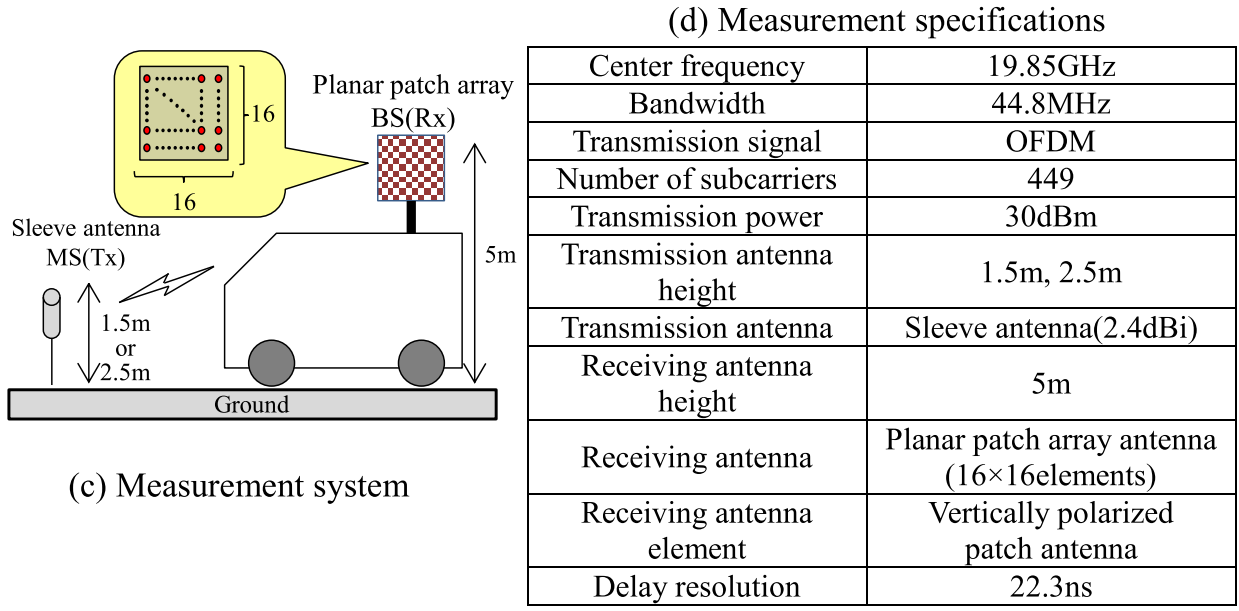

Fig. 2. Measurement environment and specifications

subcarrier in the frequency domain) acquired by each element. An average delay profile was calculated by averaging the delay profiles obtained by the respective antenna elements. The largest delay time of the measured delay profile is $10000 \mathrm{~ns}$. The largest delay time was set to $2209 \mathrm{~ns}$ in the analysis, the median value after that time (between 2232 and $10000 \mathrm{~ns}$ ) was found, and the value beyond $5 \mathrm{~dB}$ from the median value was taken as the noise floor. All values below the noise floor were set to 0 . The delay profile obtained by the process was used for learning.

\section{Comparison of measured results and estimated results}

In the neural network model [8], the number of hidden layers is 2, the activation function is sigmoid function, the loss function is binary cross-entropy, and the optimization algorithm is Adam. The number of neurons in the input layer is 9, the number of neurons in the output layer is 100 , the number of neurons in the hidden layer is 200 and 150, and the dropout rate is 0.2. Here, the loss function is a function that represents the magnitude of the difference between the estimation 


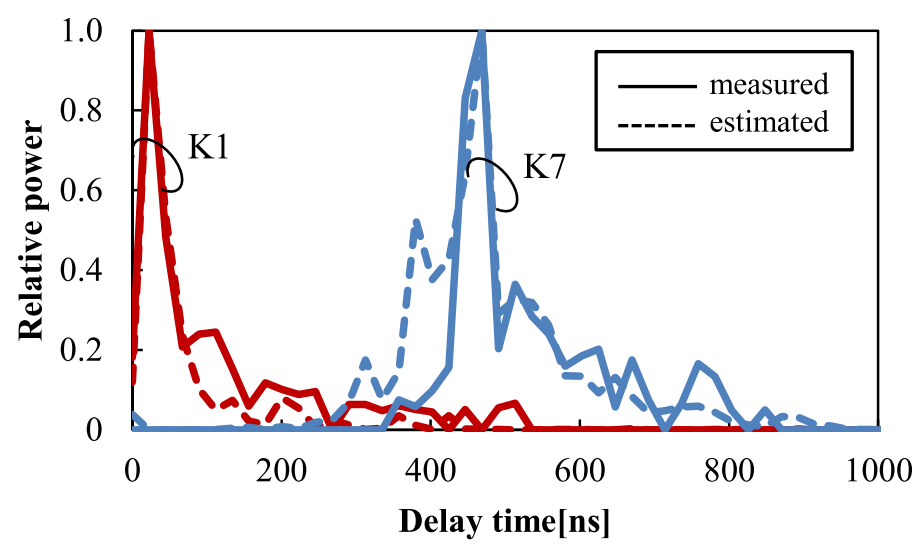

(a) Example of measured and estimated results in Kayaba

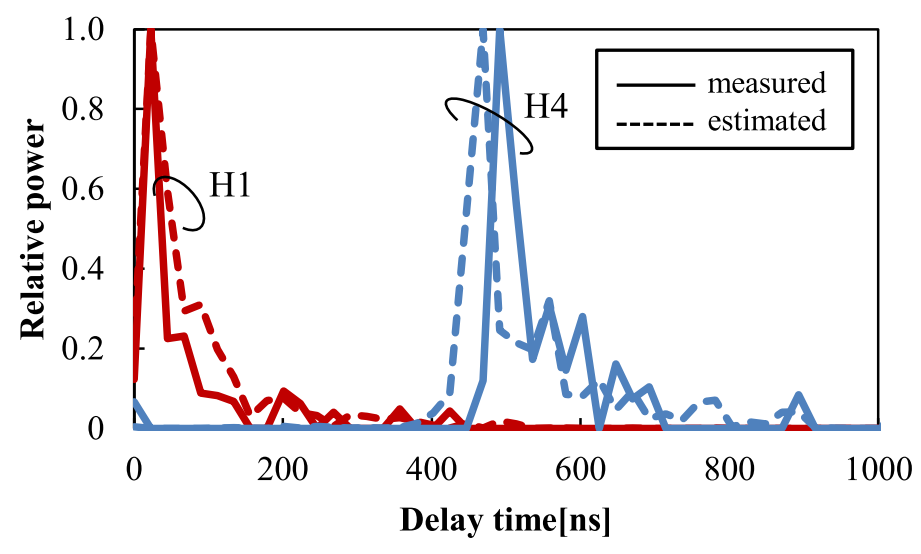

(b) Example of measured and estimated results in Hatchobori

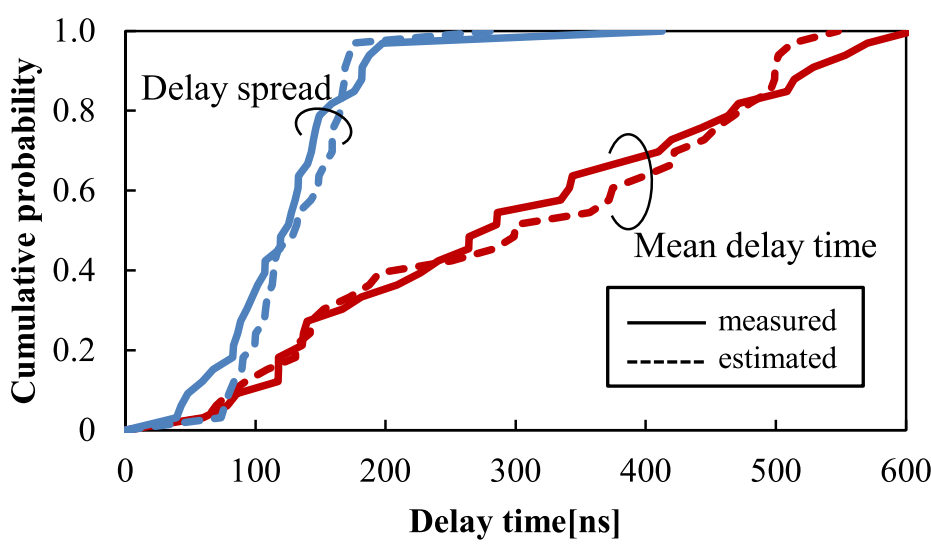

(c) CDF of mean delay time and delay spread

Fig. 3. Comparison of measurement and estimation results

and the actual value, and evaluates the estimation accuracy of the model. The optimization algorithm is an algorithm for updating the weight so that the value obtained by the loss function is minimized. By repeatedly updating the weights, the delay time estimation model is constructed. Since the constructed delay time estimation model has 100 delay bins with delay resolution of $22.3 \mathrm{~ns}$, the largest delay time that can be estimated is 2209 ns. 
Figure 3 (a) shows an example of the measured and estimated results when the transmitting station (height $1.5 \mathrm{~m}$ ) is installed at $\mathrm{K} 1$ and $\mathrm{K} 7$ as verification data. In order to show that the estimation model can estimate the delay time of unmeasured points, those used as training data is not used for verification data. Here, the data for verification (the number of data: 30) was set to one of the transmitting stations, and the data for learning was data different from the data for the verification (number of data: 960). The measurement result when a transmitting station with a height of 1.5 $\mathrm{m}$ is installed in $\mathrm{K} 1$ shows that the peak of the first delayed wave of the estimated result matches the measured result, and the delay time can be estimated with high accuracy. In the case of $\mathrm{K} 7$, although the peak positions are partially different, many peaks coincided. Figure 3 (b) shows an example of the measured and estimated results when the transmitting station (height $2.5 \mathrm{~m}$ ) is installed at $\mathrm{H} 1$ and $\mathrm{H} 4$ in Hatchobori. Except for the difference in the peaks of the first delayed wave between the estimated and measured results for $\mathrm{H} 4$, the peaks coincide in many parts. So it can be said that the delay time estimation is possible by the proposed method.

Figure 3 (c) shows the cumulative distribution function of the mean delay time and delay spread at all transmitting stations. It is a combination of multiple cases. Different transmitters are used as verification data for each case, and are separated from the training data. In the learning for constructing the delay time estimation model, the noise floor processed delay profile was used. Therefore, the delay profile output by this model is the noise-floor-processed one. When calculating the average delay time and delay spread, the output delay profile was used without the noise floor processing. It can be confirmed that the distribution of the measured results and the estimated results are almost the same for both the mean delay time and the delay spread. The median of the measured delay spread is $125.52 \mathrm{~ns}$, and the median of the estimated delay spread is $131.68 \mathrm{~ns}$. These differences are as small as $6.16 \mathrm{~ns}$. This suggests that the propagation delay time can be estimated not only direct waves but also delayed waves by the proposed method.

\section{Conclusion}

A simple propagation delay estimation method by neural network using urban structure parameters as input was proposed. It was shown that the proposed method can accurately estimate the propagation delay time without considering moving objects and trees. 